\title{
Metformina e intervenciones sobre el estilo de vida para paliar el aumento de peso inducido por antipsicóticos
}

Metformin and life style interventions to treat anti-psychotic induced weight gain

Wu R y col. JAMA. 2008;299(2):185-93

\section{Objetivo}

Evaluar la eficacia de las intervenciones sobre el estilo de vida (IEV) y la metformina para paliar el aumento de peso y las alteraciones en la sensibilidad insulínica inducidos por antipsicóticos.

\section{Diseño, lugar y pacientes}

Ensayo clínico aleatorizado doble ciego de 12 semanas de duración que incluyó 128 pacientes de 18 a 45 años con diagnóstico de esquizofrenia con su primer episodio psicótico estabilizado y cuyo peso hubiera subido más del $10 \%$ durante el primer año de tratamiento antipsicótico. Tomó lugar en una clínica de tratamiento ambulatorio de pacientes con esquizofrénia en China.

\section{Intervención y medición de resultados principales}

Fueron asignados al azar 32 pacientes a cada a uno de los siguientes grupos: 1) sólo metformina $(750 \mathrm{mg} /$ día en tres tomas diarias); 2) sólo placebo; 3) IEV (psicoeducación, dieta y sesiones de ejercicio) más metformina con la misma posología; 4) IEV más placebo. El análisis se realizó separadamente para los pacientes que completaron el tratamiento y para todos los aleatorizados con al menos una visita de seguimiento.

Resultados principales

118 pacientes completaron las 12 semanas del estudio. El grupo asignado a IEV más metformina tuvo resultados clínicos significativamente mejores que el resto. Se resumen en la tabla 1.

Tabla 1: efectos de la metformina y las intervenciones sobre el estilo de vida en pacientes con esquizofrenia.

\begin{tabular}{|c|c|c|c|c|}
\hline \multirow{3}{*}{$\begin{array}{l}\text { Intervenciones sobre } \\
\text { el estilo de vida (IEV) }\end{array}$} & \multirow{2}{*}{\multicolumn{4}{|c|}{ Diferencia media respecto al basal (IE) }} \\
\hline & & & & \\
\hline & on IEV & V V & n IEV & Sin IE \\
\hline rpora & $\begin{array}{c}-1,8 \\
(-2,3 \text { a }-1,3)\end{array}$ & $\begin{array}{c}-1,2 \\
(-1,5 \text { a }-0,9)\end{array}$ & $\begin{array}{c}-0,5 \\
(-0,8 \text { a }-0,3)\end{array}$ & $(0,9$ a 1,5$)$ \\
\hline rcunf & 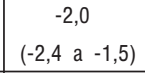 & 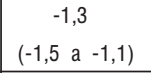 & $\begin{array}{c}0,1 \\
(-0,5 \text { a } 0,7)\end{array}$ & $\begin{array}{c}2,2 \\
(1,7 \text { a } 2,8)\end{array}$ \\
\hline $\begin{array}{l}\text { sulinemia en ayur } \\
\mathrm{UI} / \mathrm{mL} \text { ) }\end{array}$ & $\begin{array}{c}-13,9 \\
(-17,1 \text { a }-10,8)\end{array}$ & $\begin{array}{c}-12,7 \\
(-15,3 \text { a }-10,2)\end{array}$ & $\begin{array}{c}-2,7 \\
(-4,3 \text { a }-1,1)\end{array}$ & $\begin{array}{c}2,1 \\
(1,0 \text { a } 3,2)\end{array}$ \\
\hline $\begin{array}{l}\text { ice de } \\
\text { ulínica }\end{array}$ & $\begin{array}{c}-3,6 \\
(-4,5 \text { a }-2,7) \\
\end{array}$ & $\begin{array}{c}-3,5 \\
(-4,4 \text { a } \quad-2,7) \\
\end{array}$ & $\begin{array}{c}-1,0 \\
(-1,5 \text { a }-0,5) \\
\end{array}$ & $\begin{array}{c}0,4 \\
(0,1 \text { a } 0,7) \\
\end{array}$ \\
\hline
\end{tabular}

Conclusiones

Las IEV y la metformina, solas y juntas, fueron eficaces para tratar el aumento de peso inducido por antipsicóticos.

Palabras clave: Metformina, antipsicóticos, aumento de peso, intervenciones sobre el estilo de vida, ensayo randomizado controlado.

Key words: metformin, weight increase, life style interventions, randomized controlled trial.

Fuente de financiamiento: Ministerio Chino de Ciencias y Tecnología.

\section{Comentario}

El aumento de peso inducido por antipsicóticos atípicos genera complicaciones ${ }^{1,2,3,4}$ y afecta la adherencia al tratamiento ${ }^{5}$. Sibutramina ${ }^{6}$, amantadina ${ }^{7}$, topiramato ${ }^{8}$, ranitidina ${ }^{9}$, nizatidina y reboxetina $^{10}$ serían eficaces para controlarlo, pero no han demostrado efectividad sobre la sensibilidad a la insulina ${ }^{11}$. Las IEV también habían demostrado efectividad para controlar el peso $^{12}$, pero son difíciles de instituir en estas poblaciones. Respecto de la metformina, ya había demostrado reducir la ganancia de peso ${ }^{11}$, lo que es reforzado por el estudio que hemos resumido. Sin embargo duró solo 12 semanas y las dosis de antipsicóticos usadas fueron bajas ( $6 \mathrm{mg} / \mathrm{día}$ de olanzapina y $3 \mathrm{mg} /$ día de risperidona).

\section{Conclusiones del comentador}

Hasta que contemos con más ensayos controlados a largo plazo podemos seleccionar algunas de las estrategias disponibles de acuerdo a las características de cada paciente, sus disfunciones metabólicas, y su tolerancia a cada fármaco.

Sebastián Cukier [ Hospital Tobar García e Instituto FLENI, Escobar. sebastiancukier@ hotmail.com ]

Cukier S. Metformina e intervenciones sobre el estilo de vida para paliar el aumento de peso inducido por antipsicóticos. Evid. actual. práct. ambul; 11(5): 138, Sep-oct.2008. Comentado de: Wu R y col. Lifestyle intervention and metformin for treatment of antipsychotic-induced weight gain: a randomized controlled trial. JAMA. 2008; 299(2):185-93. PMID: 18182600.

\section{Referencia}

1. Mackin P y col. Prevalence of obesity, glucosa homeostasis disorders and metabolic síndrome in psychiatric patients taking typical or atypical antipsychotic drugs: a crosssectional study. Diabetología 2005; 48:215-221

2. Henderson D y col. Glucose metabolism in patients with schizophrenia treated with atypical antipsychotic agents: a frequently sampled intravenous glucose tolerance test and minimal model analysis. Arch Gen Psychiatry 2005;65:19-28

3. Alder $\mathrm{M}$ y col. Metabolic dysregulation with atypical antipsychotics occurs in the absence of underlying disease: a placebo-controlled study of olanzapina and risperidona in dogs. Diabetes 2005; 54:862-871

4. Kelly D y col. Weight gain in adolescents treated with risperidone and conventional antipsychotics over six Months. J Child Adolesc Psychopharmacol 1998; 8:151-159 5. Lieberman J y col. Effectiveness of antipsychotic drugs in patients with chronic schizophrenia. N Engl J Med 2005; 353:1209-1223

6. Henderson D col. A double-blind placebo-controlled trial with sibutramine for olanzapina-associated weight gain. Am J Psychiatry 2005; 163:954-962

7. Graham K y col. Double-blind, placebo-controlled investigation of amantadine for weight loss in subjects who gained weight with olanzapine. Am J Psychiatry 2005; 162:1744-6

8. Canitano R. Clinical experience with topiramate to counteract neuroleptic induced weight gain in 10 individuals with autistic spectrum disorders. Brain Dev 2005; 27:228-232

9. López-Mato A y col. [Randomized, open label study on the use of ranitidine at different doses for the management of weight gain associated with olanzapine administration].Vertex. 2003 Jun-Aug;14(52):85-96

10. Baptista Ty col.Pharmacological management of atypical antipsychotic-induced weight gain. CNS Drugs. 2008; 22(6):477-95

11. Klein D y col. A randomized, Double-Blind, Placebo-Controlled Trial of Metformin Treatment of Weight Gain Associated with initiation of atypical antipsychotic therapy in children and adolescents. Am J Psychiatry 2006; 163(12): 2072-2080

12. Diabetes Prevention Program Research Group: Reduction in the incidence of type 2 diabetes with lifestyle intervention or metformin. N Engl J Med 2002; $346: 393-403$ 